\title{
CROMATISMO Y MORFOMETRÍA DE NINFAS DE LA LANGOSTA CENTROAMERICANA (SCHISTOCERCA PICEIFRONS PICEIFRONS WALKER, 1870) EN EL SUR DE TAMAULIPAS, MÉXICO
}

\section{Ángel Adalberto díAZ-SÁNCHEZ, ${ }^{1}$ Ludivina BARRIENTOS-LOZANO, ${ }^{1}$ Pedro ALMAGUER-SIERRA ${ }^{1}$ Y FIDEL BLANCO-MACÍAS ${ }^{2}$}

\author{
${ }^{1}$ División de Estudios de Posgrado e Investigación. Instituto Tecnológico de Ciudad. Victoria. Blvd. Emilio Portes \\ Gil No. 1301. C.P. 87010. Ciudad. Victoria, Tamaulipas, México. \\ ${ }^{2}$ Universidad Autónoma Chapingo, Centro Regional Universitario Centro-Norte, Calle Cruz del Sur No. 100, Col. \\ Constelación, Apdo. Postal 196, CP 98085, El Orito, Zacatecas, México. <ludivinab@yahoo.com> \\ Recibido: 20/03/2015; aceptado: 09/06/2015
}

\begin{abstract}
Díaz-Sánchez, A. A., Barrientos-Lozano, L., Almaguer-Sierra, P. \& Blanco-Macías, F. 2015. Cromatismo y morfometría de ninfas de la Langosta Centroamericana (Schistocerca piceifrons piceifrons Walker, 1870) en el sur de Tamaulipas, México. Acta Zoológica Mexicana (n. s.), 31(2): 298-305.
\end{abstract}

RESUMEN. La Langosta Centroamericana (Schistocerca piceifrons piceifrons) es una de las plagas más importantes en México y Centro América. Es un insecto polífago que causa grandes daños económicos a cultivos agrícolas. Presenta dos fases extremas: solitaria y gregaria. Aquí se documenta la variación cromática y morfométrica de poblaciones de la Langosta Centroamericana (S. p. piceifrons) en el sur de Tamaulipas, México. El análisis estadístico de correspondencia demostró la asociación de la densidad de población con el cromatismo de las ninfas. Ninfas con cromatismo rosa fueron asociadas a densidades de 2000 ninfas ha-1 ${ }^{-1}$ más, ninfas con cromatismo verde fueron asocia-

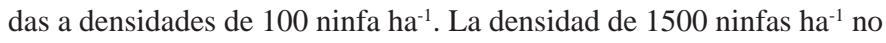
presentó una asociación definida, a esta densidad pueden encontrarse ninfas amarillas, rosa y verdes en una misma población. En el análisis de funciones discriminantes lineales se obtuvieron dos caracteres morfométricos que separan $100 \%$ ninfas de $6^{\circ}$ estadio de la Langosta Centroamericana según su cromatismo, estos caracteres son longitud del cuerpo (LCU) y ancho del pronoto (AP). Las poblaciones de $S$. $p$. piceifrons están en constante transición de fase y pueden diferenciarse en su cromatismo y morfometría.

Palabras clave: Langosta centroamericana, fases, densidad poblacional.

\section{INTRODUCCIÓN}

Entre las especies del género Schistocerca Stål, 1873 (Acrididae: Cyrtacanthacridinae) que ocurren en México, Schistocerca piceifrons piceifrons es la única capaz de gregarizar y emigrar grandes distancias; presenta además el fenómeno de fases y polifenismo, exhibiendo dos formas extremas llamadas fase solitaria y fase gregaria (Barrientos-Lozano 2001). Ésta es una de las plagas más importantes en México y Centro América (Barrientos-Lozano 2003). La presencia de la Langosta Centroamericana (S. p. piceifrons) en la región sur del estado de Tamaulipas, México es un problema antiguo, Trujillo (1975)
Díaz-Sánchez, A. A., Barrientos-Lozano, L., Almaguer-Sierra, P. \& Blanco-Macías, F. 2015. Chromatism and morphometrics of nymphs of the Central American Locust (Schistocerca piceifrons piceifrons Walker, 1870) in South Tamaulipas, Mexico. Acta Zoológica Mexicana (n. s.), 31(2): 298-305.

ABSTRACT. The Central American Locust (Schistocerca piceifrons piceifrons) is one of the most important pests in México and Central America. It is a polyphagous insect that causes great economic damage to agricultural crops. It exhibits two extreme phases: solitarious and gregarious. Here we document chromatic and morphometric variation of the Central American Locust (S. p. piceifrons) populations in South Tamaulipas, Mexico. The statistical analysis of correspondence showed association of population density with nymphs' chromatism. Nymphs with pink chromatism were associated with population density of 2000 or more nymphs ha ${ }^{-1}$; nymphs with green chromatism were associated with density of 100 nymphs ha-1 . Population density of 1500 nymphs ha ${ }^{-1}$ did not show a definite association; at this density yellow, pink or green nymphs may coexist in the same population. The linear discriminant functions analysis provided two morphometric characters that separate $100 \% 6^{\text {th }}$ instar nymphs of the Central American Locust according to their chromatism, these characters are: body length (LCU) and pronotum width (AP). Populations of the Central American Locust are in constant phase transition and may be differentiated on both, chromatism and morphometry.

Key words: Central American Locust, Phases, Population density.

reporta el control de mangas de la Langosta en Ciudad Mante, Tamaulipas en mayo de 1964. A la fecha se siguen haciendo operaciones de control oportunas; sin embargo, cuando estas acciones se descuidan, aunado a condiciones climáticas favorables, las poblaciones de $S$. $p$. piceifrons incrementan rápidamente su densidad de población, y es cuando causan grandes daños económicos (Barrientos-Lozano et al. 1992). Esta especie se alimenta tanto de vegetación verde como seca, prefiriendo los cultivos agrícolas (maíz, soya, caña de azúcar, cacahuate, sorgo, agave) a los bosques y pastos. No obstante, es capaz de alimentarse de cualquier tipo de vegetación, si no encuentra el alimento preferido (Ávila-Valdez et al. 2004). En 
la Planicie Huasteca se le ha observado alimentándose de diversas plantas y arbustos silvestres como mezquite (Prosopis juliflora), huizache (Acacia farmesiana), guazima (Guazuma ulmifolia), choveno (Mimosa pigra), guamúchil (Phitecolobium dulce), tronadora (Crotalaria sp.) entre otras, y de diferentes pastos como guinea (Panicun maximun), pangola (Digitaria decumbens), pasto bermuda (Cynodon dactilon) y pasto estrella (Cynodon plectostachyus) (Garza 2005). La Langosta Centroamericana presenta variaciones cromáticas y morfométricas desde que inicia su transformación hasta su completa evolución fásica. El color de estos insectos es críptico a baja densidad (fase solitaria), pero cuando las condiciones le son favorables se incrementa la densidad de población y las ninfas adquieren colores llamativos (fase gregaria) (Barrientos-Lozano et al. 1992). El índice de densidad que induce a la transformación fásica varía entre 500 y 50,000 individuos por hectárea según la especie. Por ejemplo, las primeras manifestaciones de gregarización de S. gregaria se observan a partir de 500 individuos ha-1 ${ }^{-1}$ mientras que para Locusta migratoria es de 2,000 individuos ha-1 ${ }^{-1} \mathrm{Ba}-$ rrientos-Lozano et al. 1992). En el caso de S. p. piceifrons el cromatismo ninfal es uno de los cambios más visibles, las ninfas pueden ser color verde, amarillo y rosa pálido o intenso; con maculaturas negras en todos los casos. Se considera solitaricolor a ninfas de color verde, transiticolor a las de color amarillo y gregaricolor a las de color rosa intenso con maculaturas negras (Barrientos-Lozano et al. 1992). En S. emarginata y S. gregaria los depredadores asocian esta coloración conspicua con toxicidad y por lo tanto las langostas son menos susceptibles de ser depredadas (Sword 1999; Sword et al. 2000). De acuerdo con Uvarov $(1921$; 1928) esta transformación de una fase a otra en las langostas requiere más de una generación para completarse y puede ser reversible. Sin embargo, Sword (1999) menciona que esta transición puede ocurrir en una sola generación, debido a la plasticidad fenotípica que presentan las langostas. La transformación fásica en langostas es una forma extrema de plasticidad fenotípica, las langostas solitarias (crípticas) pueden transformarse en gregarias (conspicuas) en respuesta a un aumento en la densidad de población (Song et al. 2007). Esto debido a una adaptación evolutiva a un entorno heterogéneo (Sword 2002). Las ninfas de la Langosta Centroamericana pasan por seis estadios generalmente, aunque pueden presentar hasta siete estadios (Astacio 1990); Barrientos-Lozano (2002) consigna cinco estadios en poblaciones gregarias. Ávila-Valdez et al. (2004) indican que la duración del periodo ninfal es de 30 días para la primera generación (Mayo-Julio) y 60 días para la segunda (Octubre-Noviembre). Una vez que cambian el comportamiento y el color, cambia la morfometría en la última etapa de la transformación fásica (Barrientos-Lozano et al. 1992). El primer instar en $S$. p. piceifrons mide de 6 a $7 \mathrm{~mm}$, el segundo de 10 a $14 \mathrm{~mm}$, el tercero de 16 a $18 \mathrm{~mm}$, el cuarto de 20 a 24 $\mathrm{mm}$, el quinto de 27 a $30 \mathrm{~mm}$ y el sexto de 35 a $45 \mathrm{~mm}$ (Garza 2005). Bredo (1963) mostro que el índice entre la longitud del fémur posterior y el ancho máximo de la cabeza (F/C) de $S$. $p$. piceifrons separa las poblaciones solitaria (>3.9) y gregaria (<3.9). Harvey (1983) también demostró que el índice (F/C) separa poblaciones solitarias de las gregarias. La función exacta de la plasticidad fenotípica en langostas no está clara, estudios recientes en otras especies asocian la plasticidad fenotípica con la densidad de población y sugieren que ésta podría ser adaptativa (Sword et al. 2000; Sword 2002). El presente trabajo tuvo como objetivo evaluar cambios cromáticos y morfométricos en ninfas de $S$. p. piceifrons, en el Sur de Tamaulipas.

\section{MATERIAL Y MÉTODOS}

Ninfas de la Langosta Centroamericana fueron recolectadas en el al Ejido México Libre, Municipio de Antiguo Morelos (N 2240’96” W 9906’98”) sur de Tamaulipas, de Septiembre a Noviembre de 2013. El muestreo y recolecta de ejemplares se realizó en la misma localidad, en áreas previamente ubicadas, por el personal técnico de la Junta Local de Sanidad Vegetal del Municipio de Mante, quienes verifican en forma permanente las poblaciones de la Langosta Centroamericana en el Sur de Tamaulipas. Se registraron las coordenadas geográficas; la densidad poblacional de ninfas y el tipo de vegetación. Para evaluar la densidad de población (ninfas) se utilizó el método de “conteo a la vista” (Pérez \& Sánchez 2005). Las ninfas fueron recolectadas con redes entomológicas y transportadas al Laboratorio de Ecología del Instituto Tecnológico de Ciudad Victoria en jaulas de $50 \mathrm{~cm} \times 50 \mathrm{~cm} \times 50$ $\mathrm{cm}$. Las jaulas fueron fabricadas previamente con madera y malla metálica. Las variables morfométricas que se midieron con vernier fueron: longitud del cuerpo (LCU) (desde el fastigio al ápice del abdomen); longitud de la cabeza (LCA); ancho de la cabeza (AC); longitud del pronoto (LP); ancho del pronoto (AP); longitud de las tegmina (LTEG) y longitud del fémur posterior (LFP) (Fig. 1). Se determinó el sexo, el estadio ninfal y se valoró el cromatismo (color) de las ninfas individualmente utilizándose tabla de colores.

Se obtuvo el índice F/C en ninfas de $6^{\text {to }}$ estadio, para determinar si las poblaciones son gregarias o solitarias (Bredo 1963; Harvey 1983) (Excel 2010). Se utilizaron ninfas de $6^{\text {to }}$ estadio debido a que en este se presentan los cambios a nivel morfométrico para pasar a estado adulto, además que para los análisis estadísticos se requieren 


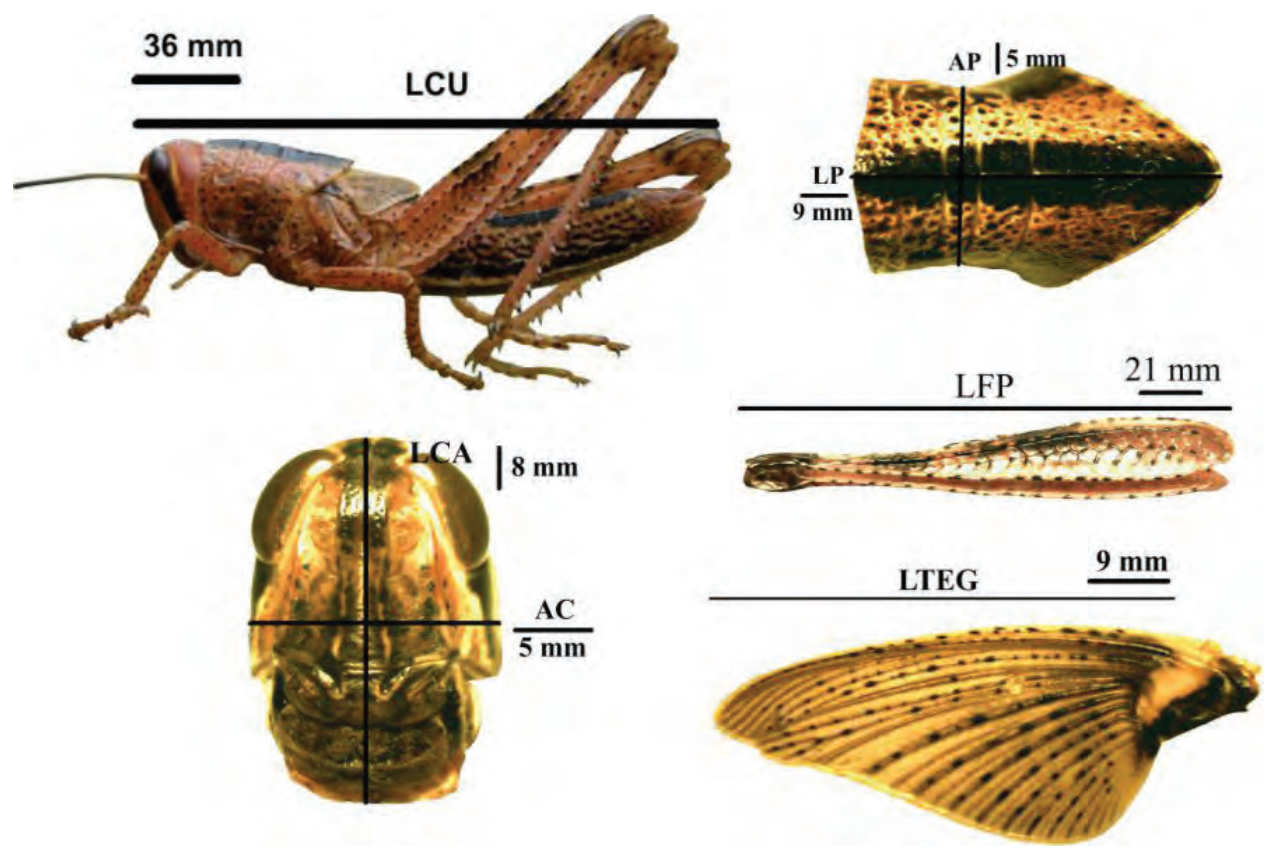

Figura 1. Variables morfométricas medidas en ninfas de la Langosta Centroamericana (S. p. piceifrons).

más de tres datos por grupo. Para determinar si existe asociación entre la densidad de población y el cromatismo de las ninfas, se aplicó un análisis de correspondencia. La manera en que se ordenaron los datos fue en forma $\mathrm{Q}$ (se ordenaron las densidades en función del cromatismo) (Legendre \& Legendre 1998) (Statistica Versión 7.0). Para evaluar diferencias significativas en la morfometría entre ninfas verdes, amarillas y rosas de $6^{\text {to }}$ estadio, se realizó un análisis de funciones discriminantes lineales (Legendre \& Legendre 1998) (Statistica Versión 7.0). Se estimó el índice de Cohen Kappa, para observar que tan bien estaban clasificados los elementos evaluados, donde valores cercanos a 0 indican que hay una mala clasificación y valores cercanos a 1 es una buena clasificación (Cohen 1960).

\section{RESULTADOS}

Entre el 5 de septiembre y el 12 de noviembre de 2013 se realizaron tres viajes al sur de Tamaulipas, para estudiar las poblaciones de $S$. $p$. piceifrons. Esta especie presenta dos generaciones al año, en consecuencia se presentan dos periodos de saltones o ninfas, uno en julio-agosto y otro en septiembre-noviembre. El 5 de septiembre, la densidad de población fue de 100 ninfas ha-1, dominando ninfas de $6^{\circ}$ estadio con cromatismo verde; para el 31 de octubre, la densidad de población se incrementó a 1500 ninfas ha-1, la mayoría de $6^{\circ}$ estadio con cromatismo verde, amarillo y rosa; para el 12 de noviembre, la densidad se incrementó a 2000 ninfas ha-1 ${ }^{-1}$ la mayoría de $6^{\circ}$ estadio y cromatismo rosa (Cuadro 1). Durante este periodo se recolectaron 341

Cuadro 1. Localidades de muestreo y colecta de ninfas de la Langosta Centroamericana (S. p. piceifrons).

\begin{tabular}{|c|c|c|c|c|}
\hline Fecha de colecta & Localidad & Coordenadas & Densidad & Tipo de Vegetación \\
\hline 5 septiembre 2013 & $\begin{array}{l}\text { Ejido México Libre, } \\
\text { Municipio Antiguo Morelos }\end{array}$ & $\begin{array}{l}\left(\mathrm{N} 22^{\circ} 40^{\prime} 9.62 ”\right. \\
\text { W9906’9.85”) }\end{array}$ & $\begin{array}{l}\text { (1 ninfa } \mathrm{m}^{-2} / 100 \text { repeticiones } \\
* 10000) 100 \text { ninfas ha-1) }\end{array}$ & $\begin{array}{l}\text { Cultivo de caña (Saccharum } \\
\text { officinarum) y pasto buffel } \\
\text { (Cenchrus ciliaris) }\end{array}$ \\
\hline 31 octubre de 2013 & $\begin{array}{l}\text { Ejido México Libre, } \\
\text { Municipio Antiguo Morelos }\end{array}$ & $\begin{array}{l}\left(\mathrm{N} 22^{\circ} 40^{\prime} 9.62 ”\right. \\
\text { W9906’9.85”) }\end{array}$ & $\begin{array}{l}\text { (15 ninfas } \mathrm{m}^{-2} / 100 \text { repeticiones } \\
* 10000) 1,500 \text { ninfas } \mathrm{ha}^{-1} \text { ) }\end{array}$ & $\begin{array}{l}\text { Soya (Glycine max), Cacahuate } \\
\text { (Arachis hypogaea) y pasto buffel } \\
\text { (Cenchrus ciliaris) }\end{array}$ \\
\hline
\end{tabular}

$\left(\mathrm{m}^{-2}\right)$ metro cuadrado y $\left(\mathrm{ha}^{-1}\right)$ hectárea 
ninfas de diferente estadio $\left(3^{\circ}, 4^{\circ}, 5^{\circ}\right.$ y $\left.6^{\circ}\right)$ con variaciones cromáticas. Las ninfas con cromatismo rosa y maculaturas negras fueron las más abundantes, recolectándose 282 individuos; enseguida las de cromatismo verde con 45 individuos y finalmente las que presentaron cromatismo amarillo con 14 individuos (Figs. 2, 3 y 4). Se midieron los caracteres morfométricos de cada individuo. Se obtuvo el promedio y la desviación estándar en los diferentes estadios ninfales según su cromatismo (Cuadros 2, 3 y 4). Se obtuvo el índice (F/C) presentando un valor de 3.81 $\mathrm{mm}$, en ninfas de $6^{\text {to }}$ estadio. Estas ninfas se encontraron asociadas a diferente tipo de vegetación como pasto buffel (Cenchrus ciliaris); huizache (Acacia farnesiana) y guasima (Guazuma ulmifolia) y cultivos de caña (Saccharum officinarum); soya (Glycine max) y cacahuate (Arachis hypogaea) (Cuadro 1).

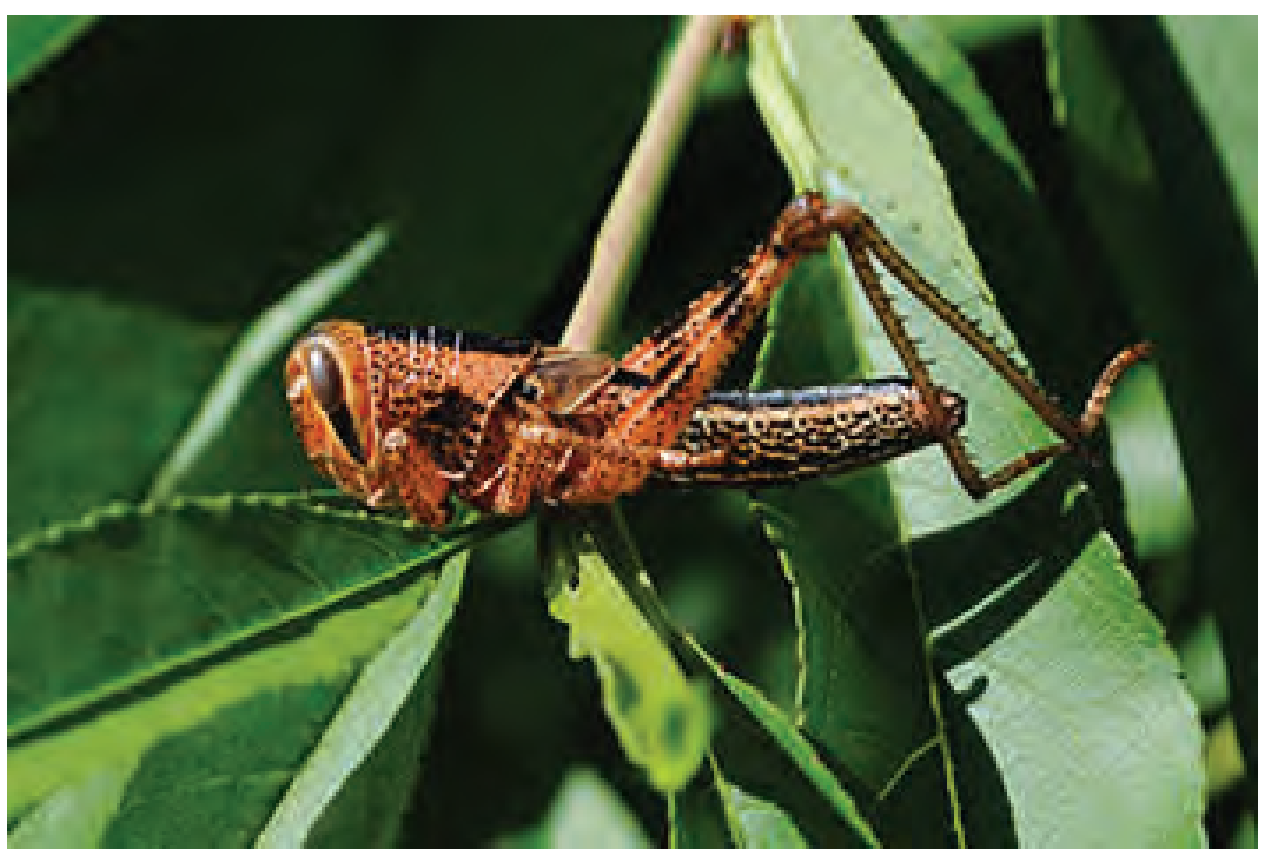

Figura 2. Langosta Centroamericana (S. p. piceifrons), ninfa con cromatismo rosa.

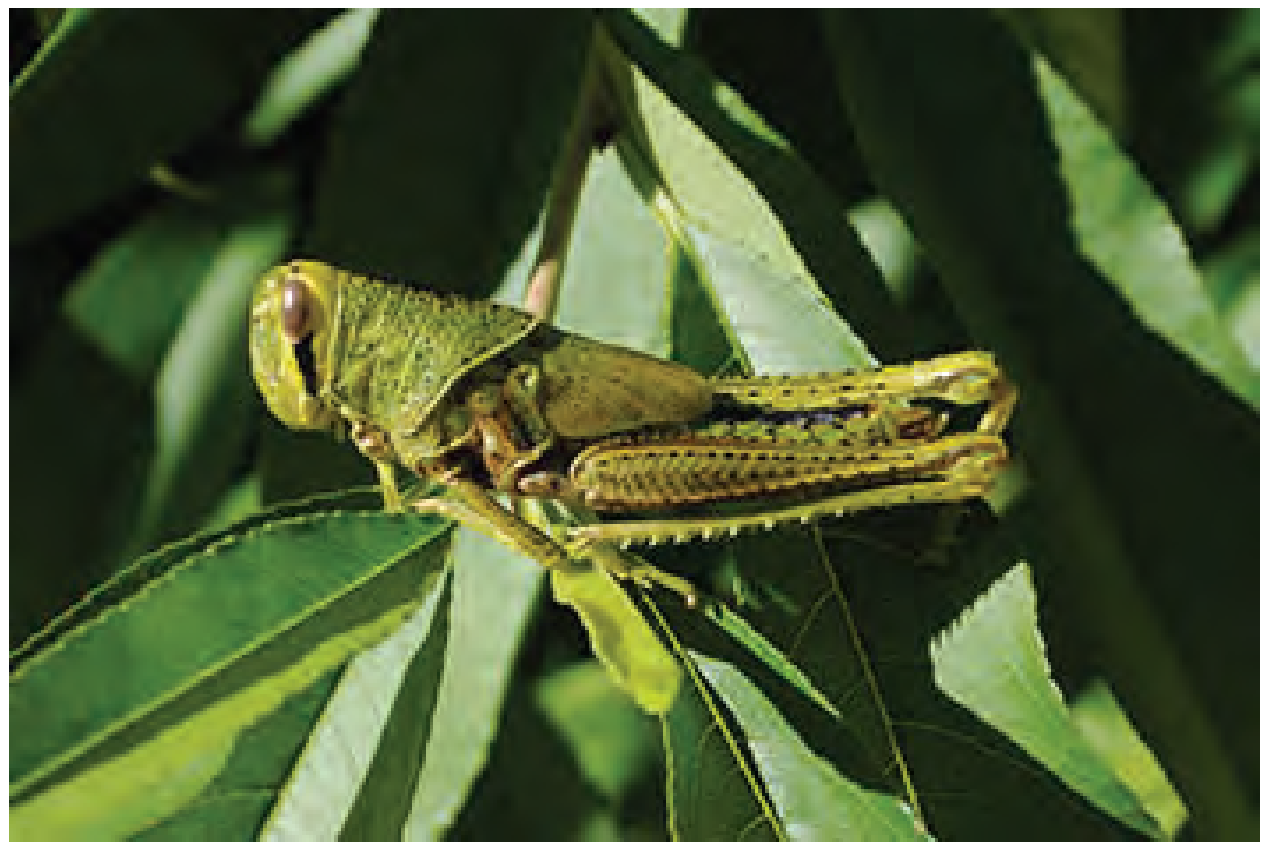

Figura 3. Langosta Centroamericana (S. p. piceifrons), ninfa con cromatismo verde. 


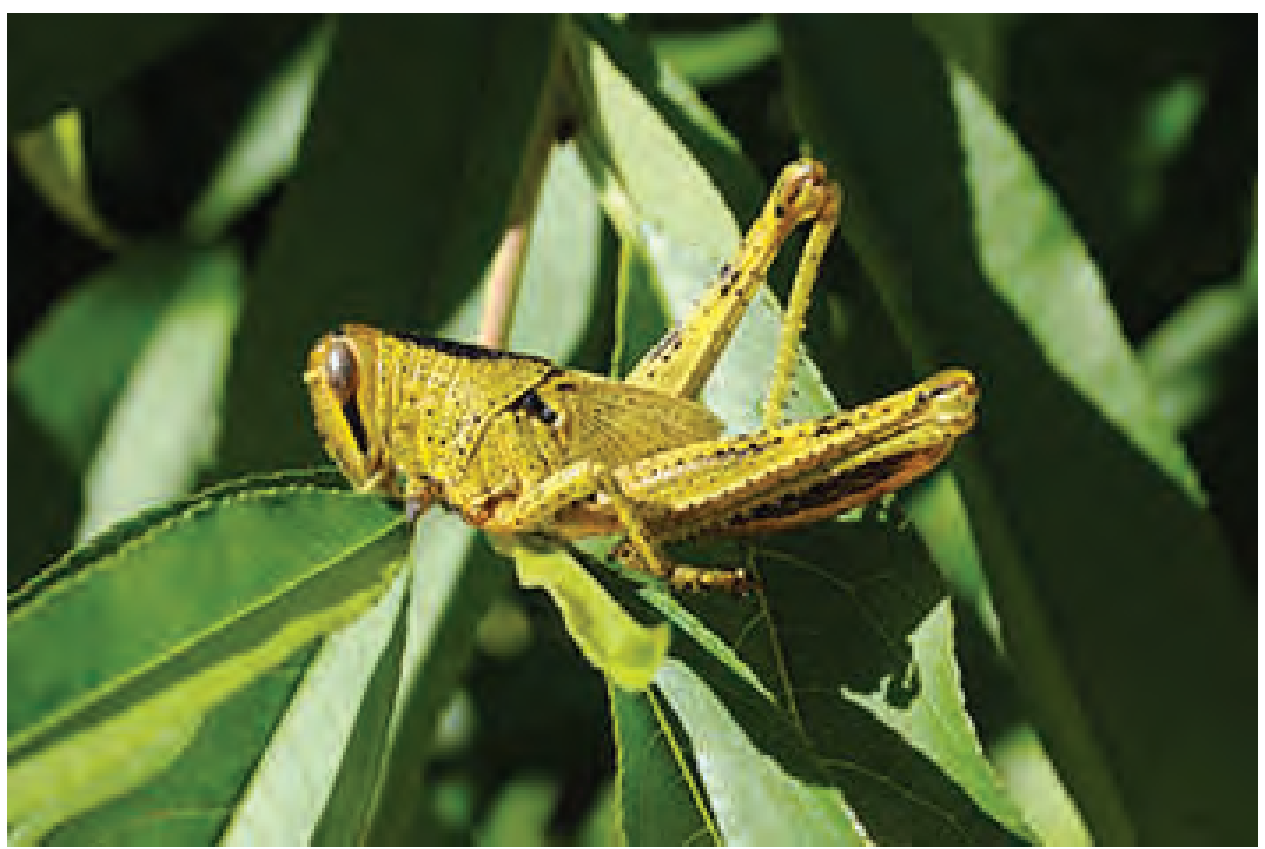

Figura 4. Langosta Centroamericana (S. p. piceifrons), ninfa con cromatismo amarillo.

Cuadro 2. Morfometría de ninfas de la Langosta Centroamericana (S. p. piceifrons) con cromatismo verde (mm). Longitud del cuerpo (LCU) (desde el fastigio al ápice del abdomen); Longitud de la cabeza (LCA); Ancho de la cabeza (AC); Longitud del pronoto (LP); Ancho del pronoto (AP); Longitud de las tegmina (LTEG) y Longitud del fémur posterior (LFP). ( $\bar{x}$ ) promedio; (Ds) desviación estándar.

\begin{tabular}{cccccccccc}
\hline Sexo & $\mathrm{n}$ & Estadio & $\begin{array}{c}\text { LCU } \\
\bar{x} \pm \mathrm{Ds}\end{array}$ & $\begin{array}{c}\text { LCA } \\
\bar{x} \pm \mathrm{Ds}\end{array}$ & $\begin{array}{c}\text { AC } \\
\bar{x} \pm \mathrm{Ds}\end{array}$ & $\begin{array}{c}\text { LP } \\
\bar{x} \pm \mathrm{Ds}\end{array}$ & $\begin{array}{c}\text { AP } \\
\bar{x} \pm \mathrm{Ds}\end{array}$ & $\begin{array}{c}\text { LTEG } \\
\bar{x} \pm \mathrm{Ds}\end{array}$ & $\begin{array}{c}\text { LFP } \\
\bar{x} \pm \mathrm{Ds}\end{array}$ \\
\hline $\mathrm{H}$ & 2 & 3 & $15 \pm 2.8$ & $7 \pm 2.8$ & $4 \pm 1.4$ & $5.5 \pm 3.5$ & $5 \pm 0$ & $2.5 \pm 2.1$ & $8 \pm 2.8$ \\
$\mathrm{H}$ & 9 & 4 & $27.4 \pm 3.4$ & $7.2 \pm 0.6$ & $4.5 \pm 0.5$ & $6.3 \pm 1.1$ & $4.5 \pm 0.7$ & $3.8 \pm 0.9$ & $15.2 \pm 1.8$ \\
$\mathrm{H}$ & 4 & 5 & $35.2 \pm 3.8$ & $9.5 \pm 0.5$ & $6 \pm 0$ & $9 \pm 0.8$ & $5.5 \pm 0.5$ & $7.5 \pm 3.5$ & $20.7 \pm 2.5$ \\
$\mathrm{H}$ & 20 & 6 & $43.8 \pm 4.8$ & $10.5 \pm 0.8$ & $6.4 \pm 0.5$ & $10.3 \pm 1.3$ & $6.7 \pm 0.6$ & $12.4 \pm 1.2$ & $24.1 \pm 2.1$ \\
$\mathrm{M}$ & 2 & 4 & $24 \pm 2.8$ & $6 \pm 1.4$ & $4.5 \pm 0.7$ & $5 \pm 1.4$ & $4 \pm 1.4$ & $3 \pm 1.4$ & $13.5 \pm 2.1$ \\
$\mathrm{M}$ & 3 & 5 & $30.3 \pm 0.7$ & $7 \pm 0$ & $4.6 \pm 0.7$ & $7 \pm 0$ & $4.3 \pm 0.7$ & $4.3 \pm 0.7$ & $16.3 \pm 0.7$ \\
$\mathrm{M}$ & 5 & 6 & $42.8 \pm 2.4$ & $9.8 \pm 0.8$ & $5.8 \pm 0.4$ & $9.8 \pm 1.3$ & $6.2 \pm 1.4$ & $10.4 \pm 1.1$ & $23.6 \pm 1.8$ \\
\hline
\end{tabular}

Cuadro 3. Morfometría de ninfas de la Langosta Centroamericana (S. p. piceifrons) con cromatismo rosa (mm). Longitud del cuerpo (LCU) (desde el fastigio al ápice del abdomen); longitud de la cabeza (LCA); ancho de la cabeza (AC); longitud del pronoto (LP); ancho del pronoto (AP); longitud de las tegmina (LTEG) y longitud del fémur posterior (LFP). ( $\bar{x}$ ) promedio; (Ds) desviación estándar.

\begin{tabular}{cccccccccc}
\hline Sexo & $\mathrm{n}$ & Estadio & $\begin{array}{c}\text { LCU } \\
\bar{x} \pm \mathrm{Ds}\end{array}$ & $\begin{array}{c}\text { LCA } \\
\bar{x} \pm \mathrm{Ds}\end{array}$ & $\begin{array}{c}\text { AC } \\
\bar{x} \pm \mathrm{Ds}\end{array}$ & $\begin{array}{c}\text { LP } \\
\bar{x} \pm \mathrm{Ds}\end{array}$ & $\begin{array}{c}\text { AP } \\
\bar{x} \pm \mathrm{Ds}\end{array}$ & $\begin{array}{c}\text { LTEG } \\
\bar{x} \pm \mathrm{Ds}\end{array}$ & $\begin{array}{c}\text { LFP } \\
\bar{x} \pm \mathrm{Ds}\end{array}$ \\
\hline $\mathrm{H}$ & 17 & 3 & $20.5 \pm 2.6$ & $5.7 \pm 0.6$ & $3.5 \pm 0.5$ & $4.8 \pm 0.9$ & $3.5 \pm 0.5$ & $1.6 \pm 0.8$ & $12 \pm 2$ \\
$\mathrm{H}$ & 67 & 4 & $26.7 \pm 3.9$ & $6.8 \pm 0.7$ & $4.2 \pm 0.5$ & $6.4 \pm 0.9$ & $4.4 \pm 0.6$ & $3.4 \pm 1.2$ & $15 \pm 1.8$ \\
$\mathrm{H}$ & 60 & 5 & $36.1 \pm 2.7$ & $8.9 \pm 0.9$ & $5.4 \pm 0.4$ & $8.7 \pm 0.9$ & $5.4 \pm 0.6$ & $9.5 \pm 1.9$ & $20.4 \pm 2.2$ \\
$\mathrm{H}$ & 75 & 6 & $41.5 \pm 3$ & $9.6 \pm 0.7$ & $6 \pm 0.6$ & $9.8 \pm 0.7$ & $5.8 \pm 0.5$ & $10.8 \pm 1$ & $23 \pm 1.6$ \\
$\mathrm{M}$ & 3 & 3 & $16 \pm 3.6$ & $5.3 \pm 0.5$ & $3.3 \pm 0.5$ & $4.3 \pm 0.5$ & $3 \pm 0$ & $1.3 \pm 0.5$ & $10.6 \pm 0.5$ \\
$\mathrm{M}$ & 5 & 4 & $24.4 \pm 4.2$ & $6.4 \pm 0.8$ & $4.2 \pm 0.4$ & $5.8 \pm 1$ & $4 \pm 0.7$ & $2.8 \pm 0.4$ & $14 \pm 1.7$ \\
$\mathrm{M}$ & 43 & 5 & $35.3 \pm 3.2$ & $8.5 \pm 0.8$ & $5.3 \pm 0.7$ & $8.5 \pm 0.7$ & $5.3 \pm 0.7$ & $9 \pm 1.9$ & $20 \pm 1.9$ \\
$\mathrm{M}$ & 12 & 6 & $38.7 \pm 3.6$ & $8.7 \pm 0.4$ & $5.4 \pm 0.5$ & $8.9 \pm 0.6$ & $5.3 \pm 0.4$ & $9.7 \pm 0.6$ & $20.7 \pm 0.7$ \\
\hline
\end{tabular}


Cuadro 4. Morfometría de ninfas de la Langosta Centroamericana (S. p. piceifrons) con cromatismo amarillo (mm). Longitud del cuerpo (LCU) (desde el fastigio al ápice del abdomen); Longitud de la cabeza (LCA); Ancho de la cabeza (AC); Longitud del pronoto (LP); Ancho del pronoto (AP); Longitud de las tegmina (LTEG) y Longitud del fémur posterior (LFP). ( $\bar{x}$ ) promedio; (Ds) desviación estándar.

\begin{tabular}{cccccccccc}
\hline Sexo & $\mathrm{n}$ & Estadio & $\begin{array}{c}\text { LCU } \\
\bar{x} \pm \mathrm{Ds}\end{array}$ & $\begin{array}{c}\text { LCA } \\
\bar{x} \pm \mathrm{Ds}\end{array}$ & $\begin{array}{c}\text { AC } \\
\bar{x} \pm \mathrm{Ds}\end{array}$ & $\begin{array}{c}\text { LP } \\
\bar{x} \pm \mathrm{Ds}\end{array}$ & $\begin{array}{c}\text { AP } \\
\bar{x} \pm \mathrm{Ds}\end{array}$ & $\begin{array}{c}\text { LTEG } \\
\bar{x} \pm \mathrm{Ds}\end{array}$ & $\begin{array}{c}\text { LFP } \\
\bar{x} \pm \mathrm{Ds}\end{array}$ \\
\hline $\mathrm{H}$ & 2 & 4 & $20 \pm 2.8$ & $6.5 \pm 0.7$ & $4 \pm 1.4$ & $4.5 \pm 0.7$ & $4 \pm 1.4$ & $2.5 \pm 0.7$ & $12.5 \pm 3.5$ \\
$\mathrm{H}$ & 2 & 5 & $36.5 \pm 2.1$ & $8 \pm 0.7$ & $5.5 \pm 0$ & $8.5 \pm 0.7$ & $5 \pm 0.7$ & $5.5 \pm 0.7$ & $17.5 \pm 0.7$ \\
$\mathrm{H}$ & 5 & 6 & $39.8 \pm 2.3$ & $10.2 \pm 0.4$ & $6.2 \pm 0.4$ & $10 \pm 0.7$ & $6.2 \pm 0.4$ & $10.8 \pm 1.3$ & $23.2 \pm 2.1$ \\
$\mathrm{M}$ & 2 & 5 & $32.5 \pm 0.7$ & $7 \pm 1.4$ & $4 \pm 1.4$ & $6 \pm 1.4$ & $3.5 \pm 0.7$ & $4.5 \pm 0.7$ & $15.5 \pm 3.5$ \\
$\mathrm{M}$ & 3 & 6 & $38.3 \pm 4.7$ & $9.6 \pm 1.1$ & $6 \pm 1$ & $10 \pm 1$ & $6 \pm 0$ & $10.3 \pm 1.1$ & $22 \pm 1.7$ \\
\hline
\end{tabular}

El análisis de correspondencia $\left(\chi^{2}=166.36\right.$, g.l $=4$, $p=0.001)$ mostró que existe asociación significativa de la densidad de población con respecto al cromatismo que presentan las ninfas (Significancia $p<0.05$ ). La primera dimensión aporta la mayor variación con 91.66 \%. Las ninfas con cromatismo rosa fueron asociadas a densidades de 20 ninfas $\mathrm{m}^{-2}$ (Densidad 3), en tanto que las ninfas con cromatismo verde y amarillo fueron asociadas a la densidad de 1 ninfa $\mathrm{m}^{-2}$ (Densidad 1). La densidad de 15 ninfas $\mathrm{m}^{-2}$ (Densidad 2) no presentó una asociación definida, a esta densidad se pueden encontrar ninfas amarillas, rosa y verdes en una misma población (Fig. 5).

El análisis de funciones discriminantes lineales aportó diferencias significativas en el cromatismo de las ninfas del $6^{\circ}$ estadio Wilks' $\lambda$ : 0.241, $\mathrm{F}_{(6,42)}=7.242, p=0.001$. Las variables morfométricas consideradas en el modelo fueron; LCU (Wilks' $\lambda$ : 0.484, $\mathrm{F}_{(2,21)}=10.544, p=0.001$ ), AP (Wilks' $\lambda: 0.414, \mathrm{~F}_{(2,21)}=7.521, p=0.003$ ). Las ninfas con cromatismo verde presentaron una variación significativamente diferente a las ninfas con cromatismo rosa y amarillo $(p<0.05)$ (Cuadro 5).

La primera raíz canónica representó el 94 \% y la segunda el $6 \%$ de la variación entre los grupos. La estructura de factores que mejor representa la variación entre

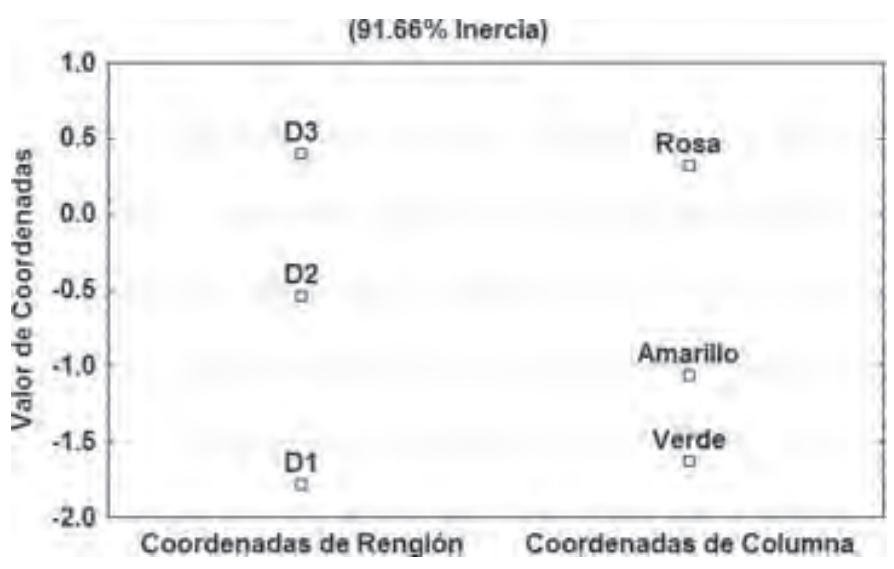

Figura 5. Análisis de correspondencia en una dimensión, de tres densidades de población de ninfas de la Langosta Centroamericana (S. p. piceifrons) en función de su cromatismo. (D1) densidad uno, (D2) densidad dos y (D3) densidad tres. el cromatismo de ninfas de $6^{\text {to }}$ estadio para las dos primeras raíces indica que las variables morfométricas que representan mejor la variación en el cromatismo fueron longitud del cuerpo (LCU) para la primer raíz y ancho del pronoto (AP) para la segunda raíz. Se muestra la posición de los centroides en los dos ejes discriminantes. El primer eje discriminante muestra las ninfas con cromatismo verde las cuales presentan LCU por encima de la media, en cuanto a las ninfas con cromatismo rosa y amarillo LCU se encuentra por debajo de la media. En el segundo eje discriminante las ninfas con cromatismo amarillo presentan AP por debajo de la media, ninfas con cromatismo verde están cerca del promedio y ninfas con cromatismo rosa presentan AP por encima de la media (Fig. 6) (Cuadros 6 y 7). Estos índices morfométricos nos diferencian el cromatis-

Cuadro 5. Valores de $\mathrm{F}$ y de $p$ en el cromatismo de ninfas de $6^{\text {to }}$ estadio de la Langosta Centroamericana (S. p. piceifrons). Fcalculada tablas y $p$-valor de probabilidad.

\begin{tabular}{|c|c|c|c|}
\hline $\mathrm{F}-p$ & Rosa & Verde & Amarillo \\
\hline Rosa & & 0.001 & 0.140 \\
\hline Verde & 17.205 & & 0.001 \\
\hline Amarillo & 2.027 & 7.489 & \\
\hline
\end{tabular}

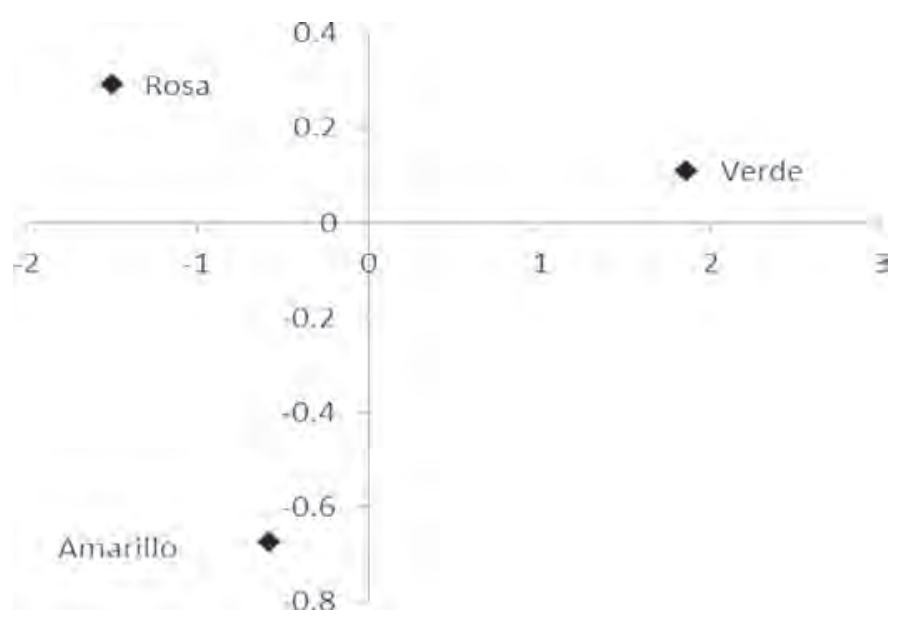

Figura 6. Centroides en los dos ejes discriminantes en el cromatismo de ninfas de $6^{\text {to }}$ estadio de la Langosta Centroamericana (S. $p$. piceifrons). 
Cuadro 6. Coordenadas de los centroides en el cromatismo de ninfas de $6^{\text {to }}$ estadio de la Langosta Centroamericana (S. p. piceifrons).

\begin{tabular}{lrr}
\hline & Raíz 1 & \multicolumn{1}{c}{ Raíz 2 } \\
\hline Rosa & -1.50333 & 0.293221 \\
Verde & 1.85424 & 0.110415 \\
Amarillo & -0.58486 & -0.672727 \\
\hline
\end{tabular}

Cuadro 7. Estructura de factores en la correlación de las variables morfométricas en el cromatismo de ninfas de $6^{\text {to }}$ estadio de la Langosta Centroamericana (S. p. piceifrons).

\begin{tabular}{lcc}
\hline & Raíz 1 & Raíz 2 \\
\hline LCU & 0.678431 & 0.557314
\end{tabular}

mo verde, rosa y amarillo en ninfas del $6^{\text {to }}$ estadio de $S$. $p$. piceifrons. Ninfas con cromatismo verde presentan LCU $(\bar{x}=45.9 \mathrm{~mm})$ y AP $(\bar{x}=6.4 \mathrm{~mm})$ mayor que las ninfas de cromatismo rosa (LCU $\bar{x}=38.2$; AP $\bar{x}=5.3 \mathrm{~mm}$ ) y amarillo (LCU $\bar{x}=38.5$; AP $\bar{x}=6.0 \mathrm{~mm}$ ), (Cuadro. 8). Ninfas con cromatismo rosa y amarillo no mostraron diferencias significativas en LCU y AP (Significancia $p<$ 0.05) (Cuadro 5).

Se obtuvo un $88.46 \%$ de elementos bien clasificados. Ninfas con cromatismo verde y amarillo fueron bien clasificadas, en tanto que tres ninfas con cromatismo rosa fueron clasificadas como ninfas de cromatismo amarillo (Cuadro. 9). En el índice de Cohen Kappa se obtuvo un valor de 0.82 , lo cual nos indica que los elementos están bien clasificados.

\section{DISCUSIÓN}

En el presente trabajo se muestra que la variación cromática, morfométrica y la transformación de fase solitaria a gregaria en la Langosta Centroamericana (S. p. piceifrons), están asociadas a la densidad de población, como lo mencionan Barrientos-Lozano et al. (1992) y Song et al. (2007). Sin embargo, aquí se aporta información sobre el índice de densación a la cual ocurre la gregarización (2000 ninfas ha-1), lo cual coindice con lo que se reporta para L. migratoria, cuya fase de gregarización inicia a una densidad de 2,000 individuos ha ${ }^{-1}$ (Barrientos-Lozano et al. 1992). Las primeras manifestaciones de gregarización en la Langosta Centroamericana se observan a partir de 1,500 ninfas ha-1, ya que a esta densidad se encontraron ninfas con cromatismo amarillo. Este cromatismo es la fase de transición de solitaria a gregaria y se le conoce como transiticolor (Barrientos-Lozano et al. 1992). Los resultados obtenidos sugieren que la transformación de fase solitaria a gregaria puede ocurrir en una sola generación, de acuerdo con Sword (2002) podría representar una adaptación evolutiva a un entorno heterogéneo. Las ninfas recolectadas representaron los estadios $3^{\circ}, 4^{\text {to }}, 5^{\text {to }}$ y $6^{\text {to }}$, lo que coincide con el reporte de Astacio (1990), quien indica que esta especie pasa por seis estadios generalmente. Respecto a su morfometría, las mediciones de LCU son muy similares a lo que reporta Garza (2005). Las poblaciones estudiadas son gregarias ya que el índice F/C presentó un valor de $3.81(\mathrm{~mm})$ en ninfas de $6^{\circ}$ estadio, como lo indican Bredo (1963) y Harvey (1983). Sin embargo, el número de estadios difiere del reportado por Barrientos-Lozano (2002), quién consigna 5 estadios para poblaciones gregarias; el número de estadios en poblaciones gregarias y solitarias se está investigando en detalle. Los caracteres morfométricos más importantes para separar ninfas de $6^{\circ}$ estadio en base a su cromatismo son longitud del cuerpo (LCU) y ancho del pronoto (AP). El índice de Cohen Kappa permitió corroborar los elementos bien clasificados en el análisis sobre cromatismo de las ninfas, con este índice se obtuvo un valor de 0.82 , lo cual nos indica una buena clasificación según Cohen (1960). Los resultados muestran que las poblaciones solitaria, gregaria y en transición de la Langosta Centroamericana pueden diferenciarse por su cromatismo y morfometría, en ninfas de $6^{\circ}$ estadio. En el sur de Tamaulipas las poblaciones de $S$. p. piceifrons están en evaluación y control permanente, lo cual contribuye a la presencia de poblaciones solitarias. Sin embargo, cuando las condiciones ambientales (temperatura, precipitación y humedad) son adecuadas las poblaciones tienden a gregarizar. La variación continua en la densidad de población induce cambios cromáticos y morfométricos, lo cual es producto de la plasticidad fenotípica de esta especie. No son claras las

Cuadro 8. Promedio y desviación estándar en milímetros de

la longitud del cuerpo (LCU) y ancho del pronoto (AP) en el cromatismos de ninfas de $6^{\text {to }}$ estadio de la Langosta Centroamericana

(S. p. piceifrons). $(\bar{x})$ promedio, (Ds) desviación estándar.

\begin{tabular}{lcc}
\hline & LCU $\bar{x} \pm$ Ds & AP $\bar{x} \pm$ Ds \\
\hline Verde & $45.9 \pm 4.04$ & $6.4 \pm 0.69$ \\
Rosa & $38.2 \pm 3.04$ & $5.3 \pm 0.48$ \\
Amarillo & $38.5 \pm 3.39$ & $6 \pm 0$ \\
\hline
\end{tabular}

Cuadro 9. Elementos bien clasificados en el cromatismo de ninfas de $6^{\text {to }}$ estadio de la Langosta Centroamericana (S. p. piceifrons).

\begin{tabular}{lccrc}
\hline & $\begin{array}{c}\text { Porcentaje } \\
\text { correcto de } \\
\text { clasificación }\end{array}$ & Rosa & Verde & Amarillo \\
\hline Rosa & 70 & 7 & 0 & 3 \\
Verde & 100 & 0 & 10 & 0 \\
Amarillo & 100 & 0 & 0 & 6 \\
Total & 88.46 & 7 & 10 & 9 \\
\hline
\end{tabular}


ventajas y/o función adaptativa que confiere a la Langosta Centroamericana la capacidad de gregarizar, es posible que el cambio en cromatismo y la gregarización representan un mecanismo de defensa contra los depredadores, como en el caso de $S$. emarginata y $S$. gregaria (Sword 1999; Sword et al. 2000). Este es un aspecto que deberá investigarse a profundidad.

AGRADECIMIENTOS. Se agradece al Ing. Hugo Nicéforo Espinosa Martínez (Junta Local de Sanidad Vegetal de ciudad Mante, Tamaulipas) por su invaluable apoyo para realizar el trabajo de campo.

\section{LITERATURA CITADA}

Astacio-Cabrera, O. 1990. La langosta Voladora o Chapulín Schistocerca piceifrons (Walker, 1870) en Centro América. Organismo Internacional Regional de Sanidad Vegetal (OIRSA). Nicaragua. $42 \mathrm{pp}$.

Ávila-Valdez, J., Barrientos-Lozano, L. \& García-Salazar, P. 2004. Biología y Comportamiento de la Langosta Centroamericana Schistocerca piceifrons piceifrons Walker. In: Manejo Integrado de la Langosta Centroamericana (Schistocerca piceifrons piceifrons Walker) y Acridoideos Plaga en América Latina. 2do. Curso Internacional. Cd. Victoria, Tamaulipas, México. Dinámica Impresa S.A de C.V. 301 pp.

Barrientos-Lozano, L., Astacio-Cabrera, O., Álvarez-Bonilla, F. \& Poot-Martínez, O. 1992. Manual técnico sobre la langosta voladora (Schistocerca piceifrons piceifrons Walker, 1870) y otros Acridoideos de Centro América y Sureste de México. FAO-OIRSA. 162 pp.

Barrientos-Lozano, L. 2001. Population Dynamics, Biology and Ecology of the Central American Locust (Schistocerca piceifrons piceifrons, Walker) in southern Mexico. Metaleptea. Eight International Meeting of the Orthopterists' Society. International Conference on Orthopteroid insects. Monpellier, France. P. 75.

Barrientos-Lozano, L. 2003. Ortópteros Plaga de México y Centro América: Guía de Campo. Cd. Victoria Tamaulipas, México. $114 \mathrm{pp}$.

Barrientos-Lozano, L. 2002. Comportamiento Gregario y Causas de Gregarización (Teoría de las Fases). In: Ecología, Manejo y Control de la Langosta Voladora (Schistocerca piceifrons piceifrons
Walker). Memorias Curso I. Internacional. Instituto Tecnológico de Cd. Victoria, Tamaulipas, México. Dinámica Impresa S.A de C.V. 232 pp.

Bredo, H. J. 1963. Rapport de mission relatifaletude du Schistocerca paranensis Burm., en Amerique Centrale, Panama et Mexique. FAO. Rome. 77 pp.

Cohen, J. 1960. A coefficient of agreement for nominal scales. Educational and Psychological Measurement, 41: 687-699.

Garza, U. E. 2005. La langosta Schistocerca piceifrons piceifrons y su manejo en la planicie Huasteca. Campo Experimental Ébano. INIFAP-CIRNE. San Luis Potosí, México. Folleto Técnico Número. 12: 23.

Harvey, A. W. 1983. Schistocerca piceifrons (Walker) (Orthoptera: Acrididae), the swarming locust of tropical America: a review. Commonwealth Agricultural Bureaux. Bulletin of Entomological. Research, 73: 171-184.

Legendre, P. \& Legendre, L. 1998. Numerical Ecology. Second English Edition. Developments in Environmental Modelling, 20. Elsevier. Amsterdam, the Netherlands. 853 pp.

Pérez, M. \& Sánchez, A. 2005. Norma Oficial Mexicana 049, Campaña Contra la Langosta en México y modificaciones a su apéndice técnico. Dirección General de Sanidad Vegetal. México D.F. 16 p

Song, H \& Wenzel, W. J. 2007. Phylogeny of bird-grasshopper subfamily Cyrtacanthacridinae (Orthoptera: Acrididae) and the evolution of locust phase polyphenism. Department of Entomology, the Ohio State University, Columbus, OH 43212, USA. The Willi Henning Society, 24: 515-542.

Sword, G. A. 2002. A role of phenotypic plasticity in the evolution of aposematism. Proceedings of the Royal Society of London B, 269: $1639-1644$.

Sword, G. A., Simpson, S. J., El Hadi, O. M. \& Wilps, H. 2000. Density dependent aposematism in the desert locust. Proceedings of the Royal Society of London B, 267: 63 -68.

Sword, G. A. 1999. Density-dependent warning coloration. Nature, 397: 217.

Trujillo, G. P. 1975. El problema de la langosta Schistocerca paranensis Burm. Sociedad de Geografía y Estadística de Baja California. Tijuana, Baja California, México. 151 p.

Uvarov, B. P. 1921. A revision of the genus Locusta L. (Pachytylus, Fieb.), with a new theory as to the periodicity and migration of locusts. Bulletin of Entomological Research, 12: 135-163.

Uvarov, B. P. 1928. Locusts and Grasshoppers. A Handbook for their Study and Control. Imperial Bureau of Entomology, London. 352 pp. 